\title{
La videograbación como recurso metacognitivo en el aprendizaje de la ciencia en educación superior
}

\section{Videotaping as a metacognitive resource in science learning in higher education}

\author{
DOI: $10.46932 / \mathrm{sfjdv2n5-093}$
}

Received in: Oct 1st, 2021

Accepted in: Dec 30th, 2021

\author{
Carlos Alfredo Damián García \\ Dr. En Investigación Educativa \\ Esc. Normal Experimental \\ "Salvador Varela Reséndiz" \\ Av. de la Normal s/n Juchipila, Zac. México \\ E-mail: cabritoreposado@gmail.com
}

\section{RESUMEN}

El objetivo de la investigación fue, determinar cómo influye la videograbación como recurso metacognitivo, en el aprendizaje de la Ciencia en Educación Superior. Se sustenta en Frade (2006) aplicando los 6 procesos mentales identificados en las interacciones. Tiene un enfoque cualitativo, donde se utilizó el método de investigación-acción bajo el modelo de Kemmis. Participaron 50 estudiantes del Primer Semestre de la Licenciatura en Educación Primaria de la Escuela Normal Experimental de Juchipila, Zacatecas, México. Se utilizó la observación participante, la técnica SQA (lo que Sé, lo que Quiero saber y lo que Aprendí), la videograbación, el guion de análisis y la triangulación de datos. Dentro de los hallazgos, se encontró que los estudiantes normalistas llegan al nivel superior con pocas estrategias de aprendizaje: el resumen, lectura individual y exposición en power point, bajo el principio del mínimo esfuerzo. Sin embargo, con la intervención de la videograbación apoyada en el análisis metacognitivo de los procesos mentales: sensación, percepción, atención, memoria, cognición y actuación, favoreció el aprendizaje autónomo aprovechando el conocimiento del contexto, diálogos, habilidades mentales, destrezas, actitudes y valores, esenciales en la formación de competencias en Educación Superior.

Palabras clave: videograbación, metacognición, procesos mentales.

\begin{abstract}
The objective of the research was to determine how video recording as a metacognitive resource influences the learning of Science in Higher Education. It is based on Frade (2006) applying the 6 mental processes identified in the interactions. It has a qualitative approach, where the action research method was used under the Kemmis model. Fifty students from the First Semester of the Primary Education Degree from the Juchipila Normal School, Zacatecas, Mexico participated. Participant observation, SQA technique (what I know, what I want to know and what I learned), video recording, analysis script and data triangulation were used. Among the findings, it was found that normal school students reach the higher level with few learning strategies: summary, individual reading and power point presentation, under the principle of minimum effort. However, with the intervention of video recording supported by the metacognitive analysis of mental processes: sensation, perception, attention, memory, cognition and action, it favored autonomous learning, taking advantage of knowledge of the context, dialogues, mental abilities, skills, attitudes and values, essential in the formation of competencies in Higher Education.
\end{abstract}

Keywords: videotaping, metacognition, mental processes. 


\section{INTRODUCCIÓN}

El presente estudio se originó en las sesiones del curso "Introducción a la naturaleza de la ciencia" SEP (2018), impartido en el ciclo escolar 2018-2019 a 2 grupos del Primer Semestre de la Licenciatura en Educación Primaria, de la Escuela Normal Experimental "Salvador Varela Reséndiz" de Juchipila, Zacatecas, México, dando un total de 50 estudiantes. Al inicio del semestre, al explorar los conocimientos previos e ir revisando los cuadernos, las participaciones, los registros de tareas, las evidencias de lectura y los productos elaborados, se pudo identificar que la mayoría de los alumnos conocen pocas estrategias de aprendizaje de la ciencia: lecturas, exposiciones, experimentos, láminas, dinámicas, observaciones, resúmenes y videos. Dan mayor prioridad al uso del resumen, la lectura individual y la exposición en power point, bajo el principio del mínimo esfuerzo; por lo tanto, no cuentan con herramientas metacognitivas que impacten en su aprendizaje.

Partiendo de dicha problemática, se revisó bibliografía e investigaciones previas, de donde se retomaron algunos elementos importantes. De acuerdo con Schneider and Lockl (2008) y Fritz, Howie and Kleitman (2010) en Bonilla and Díaz (2018), ubicamos a la metacognición en dos componentes: el primero, está relacionado con el conocimiento que tiene una persona sobre los propios procesos cognitivos (saber qué), es de naturaleza declarativa y suele ser un conocimiento relativamente estable. El segundo componente se refiere a la regulación de los procesos cognitivos (saber cómo) y está asociado a las actividades de planificación, control y evaluación. Involucra el aspecto procedimental del conocimiento y permite encadenar de forma eficaz las acciones necesarias para alcanzar una meta.

Por otro lado, se retoma la importancia que menciona Marcus (2014) de la investigación visual que, en este caso, se implementó la videograbación como un recurso que facilita a los alumnos el conocimiento y regulación de sus procesos cognitivos implicados en sus actividades de aprendizaje, enriqueciendo la interpretación de los hechos a través de la descripción, representación, reflexividad, perspectivas, etc.

De acuerdo con la Secretaría de Educación Pública (SEP, 2018) el plan de estudios de la Educación Normal en México, plantea a los docentes trabajar trasversalmente, durante la carrera profesional, la competencia genérica "Aprende de manera autónoma y muestra iniciativa para auto-regularse y fortalecer su desarrollo personal"; se enfatiza en el aprender a aprender, siendo la metacognición un elemento que puede ayudar a la formación inicial de docentes para que desarrollen competencias profesionales. "El aprendizaje de las competencias genéricas es indispensable en el contexto de la educación superior, dado que aumenta en el estudiante la probabilidad de un mayor desarrollo académico y un relativamente fácil ingreso al campo laboral" (Guevara y Guerra, 2021, p. 292).

Por lo que, la presente investigación se orienta en el desarrollo de competencias de Frade (2006), donde se considera que el ser humano aprende conocimientos, habilidades, destrezas, actitudes y valores, 
al interactuar con otro sujeto, objeto, animal u otro elemento, activando 6 procesos mentales, casi de forma simultánea:

- Sensación: el cerebro recibe las diferentes sensaciones a través de los cinco sentidos: vista, oído, gusto, tacto, olfato. Por ejemplo: veo algo.

- Percepción: el cerebro hace consciente la sensación, generalmente una a la vez, es decir, ve una moto, o bien siente calor. La percepción se basa en la diferenciación, en la discriminación de las cualidades que evoca la sensación. El frío es diferente del calor y por eso se siente. No se perciben todas las sensaciones, por ejemplo, una persona puede haberse hecho un moretón y no haberlo sentido, y cuando lo ve entonces se pregunta: ¿a qué horas me pegué?, o bien, cuando ve algo, no lo percibe hasta que lo hace consciente y dice: <<veo agua $>>$.

- Atención: se le pone atención a un solo estímulo seleccionado por la percepción, y uno se concentra en él para planear la acción y ejecutarla sobre dicho objeto. Por ejemplo: se dirige a buscar la fuga de agua, se concentra en donde está.

- Memoria: se usa para identificar qué es ese estímulo, cómo se llama. Por ejemplo: si advierte que lo que ve es agua, lo hace porque tiene guardado en su memoria que a eso se le llama agua.

- Cognición o pensamiento: entiende, conoce, analiza, sintetiza las cualidades del objeto, planea y toma una decisión con respecto al mismo. Puedo analizar que la fuga viene de la llave que está mal cerrada.

- Actuación: es el momento en que se lleva a cabo el desempeño, la actividad pensada, y por lo general tiene un inicio, un nudo y un desenlace, o sea, se planea, se ejecuta y se evalúa lo que se hizo con el objeto. Por ejemplo, cierro la llave. Tiene además una actitud: cierro la perilla con actitud impulsiva, hasta con cierto temor de que el empaque o la llave no funcione.

Por lo anterior, el objetivo central de la investigación fue determinar cómo influye la videograbación como recurso metacognitivo en el aprendizaje de las Ciencias en Educación Superior.

Supuesto: La videograbación como recurso metacognitivo promueve habilidades micrológicas y macrológicas para desarrollar competencias de manera autónoma.

\section{MATERIALES Y MÉTODO}

La presente investigación se sustenta en un enfoque cualitativo con el método de investigaciónacción bajo el modelo de Kemmis, conforme lo presenta Latorre (2005) en el siguiente esquema: 


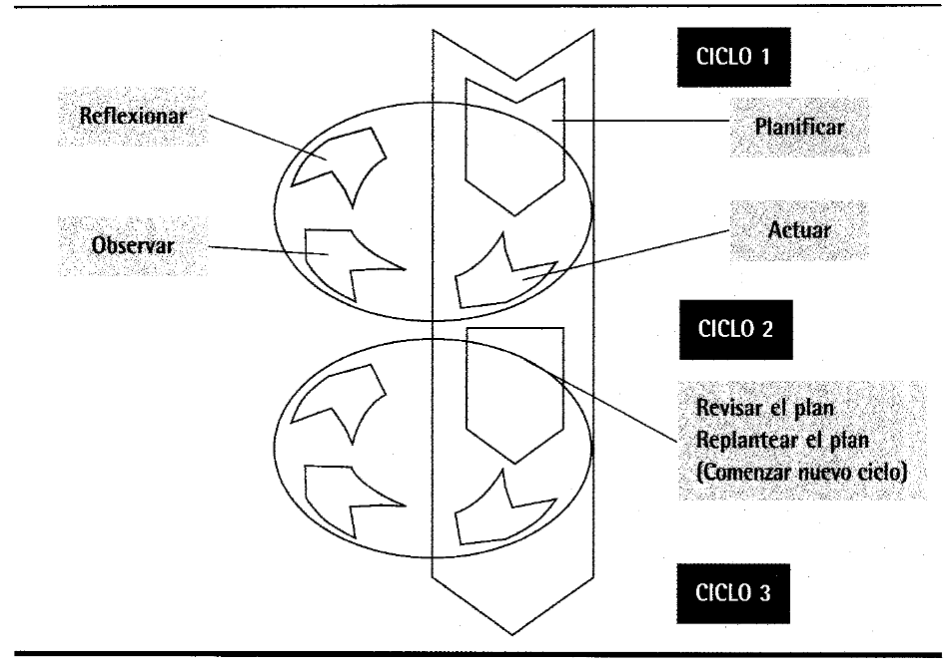

La población estuvo integrada por 50 estudiantes del Primer Semestre de la Licenciatura en Educación Primaria de la Escuela Normal Experimental de Juchipila, Zacatecas. Se utilizó la técnica observación participante, la técnica SQA (lo Que sé, lo que Quiero Saber, lo que Aprendí), la videograbación, el guion de análisis, y la triangulación de datos.

\section{RESULTADOS}

\subsection{PRIMER CICL}

Se aplicó a los alumnos la técnica SQA para explorar sus conocimientos previos respecto a la ciencia, identificándose lo siguiente:

Tabla 1 Conocimientos previos

\begin{tabular}{|c|l|l|}
\hline \multicolumn{1}{|c|}{ Lo que Sé } & \multicolumn{1}{|c|}{ Lo que me gustaría Saber } & Lo que Aprendí \\
\hline La ciencia puede ser aprendida a través de: & Aprender ciencia con estrategias & \\
- Lecturas & prácticas. & \\
- Exposiciones & & \\
- Experimentos & & \\
- Láminas & & \\
- Dinámicas & & \\
- Reservaciones & & \\
- Videos & & \\
\hline
\end{tabular}

Posteriormente, los alumnos aplicaron las estrategias de ciencia que conocían, apoyándose en sus conocimientos previos a la Educación Superior. Ejemplo: se les encargó realizar un experimento relacionado a un tema de las ciencias naturales, el cual fue aplicado en la Escuela Normal de forma individual. La mayoría de los estudiantes mencionaron el título de su experimento, los materiales que se necesitaron, ejecutaron el experimento y terminaron su participación dando una breve explicación común de los hechos. Algunos alumnos no obtuvieron resultados de su experimento, percibiéndose que lo 
Figura 2 Frecuencia de actividades en classe

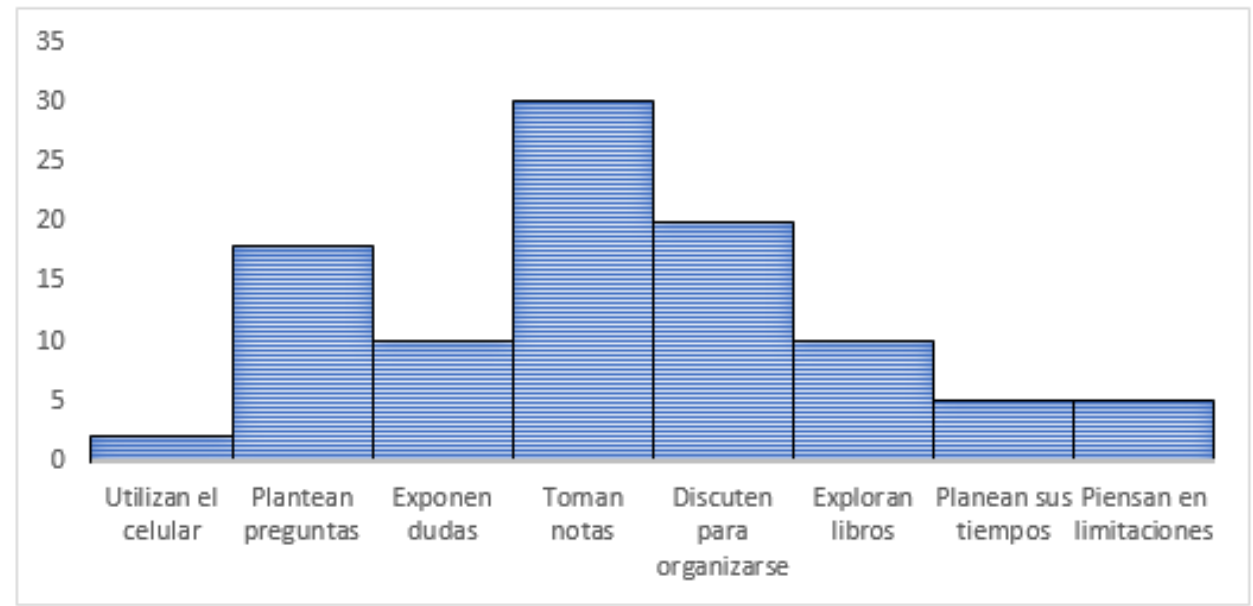

Como se puede apreciar en la figura 2, las acciones de mayor frecuencia fueron: tomar notas, discutir para organizarse y plantear preguntas; sea al maestro, compañeros o a integrantes de algún equipo. Las acciones de menor frecuencia fueron: utilizar el celular, pensar en las limitaciones y planear sus tiempos. En promedio, los alumnos consultan libros y exponen dudas. De lo anterior, se observa que la mayoría de los alumnos son dependientes y utilizan pocas actividades metacognitivas.

Así mismo, se les dio la oportunidad a los estudiantes de observar sus actividades, identificando los 6 procesos mentales que propone Frade (2006), bajo el siguiente guion de análisis:

1. Sensación: ¿Qué sentidos usaste en la actividad?

2. Percepción: ¿Qué recursos usaste en la actividad?

3. Atención: ¿En qué te concentraste?

4. Memoria de trabajo: ¿Qué es lo que usaste y para qué sirve?

5. Cognición o pensamiento: ¿Qué habilidades mentales decidiste utilizar?

6. Actuación: ¿Qué acciones motrices realizaste y qué actitudes presentaste?

Se reflexionó sobre cómo funciona nuestro cerebro cuando interactúa con el mundo; cuando una persona entra en contacto con un objeto, una cosa, dándose los procesos anteriores en nuestra mente, casi de forma simultánea. Se pudo apreciar en las diversas actividades, que los alumnos utilizaron diferentes niveles de dominio del conocimiento, conforme a la taxonomía de Bloom: Conocimiento, Comprensión, Aplicación, Análisis, Síntesis y Evaluación; denominadas por Frade (2006) como habilidades micrológicas. 


\subsection{SEGUNDO CICLO}

Los estudiantes normalistas continuaron observando sus estrategias aplicadas en las actividades académicas, percatándose de la activación de sus procesos mentales con los siguientes resultados:

Tabla 2 Tipo de actividades identificadas

\begin{tabular}{|c|c|}
\hline Proceso Mental & Resultados \\
\hline Sensación & $\begin{array}{l}\text { Actividades auditivas } 30 \% \\
\text { Actividades visuales } 35 \% \\
\text { Actividades táctiles } 20 \% \\
\text { Actividades gustativas } 5 \% \\
\text { Actividades Olfativas } 10 \%\end{array}$ \\
\hline Percepción & $\begin{array}{l}\text { Opiniones, herramientas, ambiente, explicaciones, actuación, materiales, audios, } \\
\text { computadora, celular, imágenes, pizarrón, hojas de papel, videos, maqueta, } \\
\text { diapositivas, propuestas, comentarios, reacciones, actitudes, puntos de vista, expresión } \\
\text { oral, problemas, información, sonidos, lectura, personajes, procedimientos, teorías, } \\
\text { investigaciones, comportamientos, experimentos, productos, olores, voces, grupo, } \\
\text { compañeros, respuestas, demostraciones, atención, portafolio, escucha, láminas, datos, } \\
\text { texturas, sustancias, temas, internet, apoyo, estudio, desenvolvimiento, estrategias, } \\
\text { exposiciones, instrumentos, participaciones, fruta, ingredientes, alimentos, resumen, } \\
\text { instrucciones, contenidos, sabores, risas, humo, posturas, recortes, usos, distracciones, } \\
\text { indicaciones, diseños, preguntas. }\end{array}$ \\
\hline Atención & $\begin{array}{l}\text { A lo relacionado al tema, al tipo de estrategia a desarrollar, a los conocimientos previos, } \\
\text { al conocimiento investigado, a realizar los procedimientos correctamente, al } \\
\text { comportamiento de los compañeros, a preguntar correctamente, a cuidar el vocabulario, } \\
\text { a dirigir la atención, a garantizar la observación, a la escucha, a colocar los materiales, } \\
\text { a las participaciones, a la forma de explicar y expresar la información, a las } \\
\text { investigaciones, a elaborar preguntas, a observar reacciones, a aportar argumentos, a la } \\
\text { opinión de los compañeros, al comportamiento del grupo, a la organización de los } \\
\text { equipos, a las respuestas, a las indicaciones de organización, a la claridad del tema, a } \\
\text { las preguntas que planteaban los compañeros, al uso de técnicas para explicar, a las } \\
\text { actitudes, a los procedimientos, a los errores cometidos, a las discusiones, a los } \\
\text { organizadores gráficos, a las secuencias, a los cuidados técnicos, a la creatividad de las } \\
\text { actividades, a evitar muletillas, a evitar pleonasmos, a la presentación de los demás, a } \\
\text { las interacciones de los equipos y a las dudas. }\end{array}$ \\
\hline Memoria & Identificaron a las cosas o situaciones por su nombre. \\
\hline Cognición & $\begin{array}{l}\text { Las habilidades cognitivas que más utilizaron fueron de las que integran la taxonomía } \\
\text { de Bloom; sin embargo, en el segundo ciclo, también utilizaron habilidades } \\
\text { macrológicas como toma de decisiones compleja, pensamiento crítico, pensamiento } \\
\text { autónomo, pensamiento inductivo-deductivo, pensamiento creativo y pensamiento } \\
\text { colaborativo. }\end{array}$ \\
\hline Actuacio & oría de las veces, congruente con lo pensado. \\
\hline
\end{tabular}

Por último, se les proporcionó su tabla SQA, para que anotaran lo que aprendieron en el curso, identificándose lo siguiente: 
Tabla 3 Estrategias aprendidas

\begin{tabular}{|c|c|c|}
\hline Lo que Sé & $\begin{array}{c}\text { Lo que me } \\
\text { gustaría Saber }\end{array}$ & Lo que Aprendí \\
\hline $\begin{array}{l}\text { La ciencia puede ser } \\
\text { aprendida a través de: } \\
\text { - Lecturas } \\
\text { - Exposiciones } \\
\text { - Experimentos } \\
\text { - Láminas } \\
\text { - Dinámicas } \\
\text { - Observaciones } \\
\text { - Resúmenes } \\
\text { - Videos }\end{array}$ & $\begin{array}{l}\text { Aprender } \\
\text { ciencia con } \\
\text { estrategias } \\
\text { prácticas. }\end{array}$ & $\begin{array}{l}\text { Maquetas, croquis, lluvia de ideas, tablas de comparación, } \\
\text { cuestionarios, proyectos, autoevaluaciones, manualidades, } \\
\text { investigación, materiales de reuso, periódico mural, mapas } \\
\text { mentales, dibujos, portafolios, memoramas, modelos, } \\
\text { rompecabezas, exploración, simulacros, esquemas, } \\
\text { publicaciones, cartel, material recortable, películas, internet, } \\
\text { viajes, glosarios, imágenes, trabajo colaborativo, álbum, } \\
\text { recorridos, diccionario científico, cápsulas científicas, } \\
\text { biblioteca de aula, el medio natural y social, museos, etc. }\end{array}$ \\
\hline
\end{tabular}

\section{ANÁLISIS DE RESULTADOS}

Con el uso de la técnica SQA, se promovió que los alumnos realizaran miradas retrospectivas, introspectivas y prospectivas, autorreflexionando sobre el conocimiento y la acción.

La videograbación de actividades realizadas por los estudiantes explorando sus conocimientos previos, permitió mayor autoconsciencia de lo que saben, saben hacer y saben ser, reflejado en un mayor nivel de descripción, narración, interpretación, reflexividad, perspectiva, etc.

Se observó que el canal de aprendizaje más utilizado por los alumnos fue el visual, seguido del canal auditivo y, en tercer lugar, el canal kinestésico.

Con las videograbaciones, los alumnos normalistas lograron tener mayor consciencia de la activación de los procesos mentales, identificando que la mayoría de sus acciones corresponden a las habilidades micrológicas, refiriéndose a los niveles de conocimiento de la Taxonomía de Bloom: Conocimiento, Comprensión, Aplicación, Análisis, Síntesis y Evaluación; y en menor frecuencia, utilizaron las habilidades macrológicas, refiriéndose a la toma de decisiones compleja, pensamiento crítico, pensamiento autónomo, pensamiento inductivo-deductivo, pensamiento creativo y pensamiento colaborativo.

\section{CONCLUSIONES}

La videograbación como recurso metacognitivo propició que los estudiantes normalistas se dieran cuenta de su estado inicial de conocimiento, potenciando sus capacidades a través del uso autoconsciente de los procesos mentales implicados en las interacciones, apoyado en el aprendizaje por investigación como método activo.

Lograron tener avances en dichas capacidades cognitivas, utilizando las habilidades micrológicas, consideradas por Frade (2006) como acciones básicas contenidas en la Taxonomía de Bloom y utilizando las siguientes habilidades macrológicas: 
- TOMA DE DECISIONES COMPLEJA: decide en base a ventajas y desventajas, costo y beneficio, lo que conviene y lo que no. Regula su impulso y emoción, es decir, autorregula su motivación.

- PENSAMIENTO CRÍTICO: hace comparaciones, define lo que falta, considera ventajas y desventajas, lo que vale, lo que es real y lo que no. Es un proceso racional, objetivo y emotivo que identifica relaciones, patrones, causas y efectos.

- PENSAMIENTO AUTÓNOMO: piensa de manera independiente a pesar de la presión social. Respeta a los demás.

- PENSAMIENTO INDUCTIVO-DEDUCTIVO: integra, comprueba hipótesis, es tenaz para llegar al final.

- PENSAMIENTO CREATIVO: da soluciones alternas, originales, únicas ante problemas; inventa productos, es meta-cognitivo y meta-analítico. Se autoevalúa, es osado.

- PENSAMIENTO COLABORATIVO: piensa en equipo, diseña estrategias en conjunto, negocia, controla su ego para construir junto con los demás.

Se amplió el conocimiento y aplicación de estrategias utilizadas para la enseñanza y aprendizaje de la ciencia: croquis, cambio en el tiempo, cuestionario, proyecto, diccionario científico, actividad de investigación, portafolio, instrumentos de ciencia, álbum, láminas, mapa conceptual, resumen, casos, problemas, debate, conferencia, dibujo-texto, rincón de la ciencia, visitas o recorridos, cuadro sinóptico, $\mathrm{v}$ de gowin, clase por modelización, etc.

La videograbación como recurso metacognitivo favorece el aprendizaje autónomo aprovechando el conocimiento del contexto, diálogos, habilidades mentales, destrezas, actitudes y valores, esenciales en la formación de competencias en Educación Superior. 


\section{REFERENCIAS}

Bonilla Traña, Mariel, Díaz Larenas, Claudio. La metacognición en el aprendizaje de una segunda lengua: estrategias, instrumentos y evaluación. revista educación [en linea] 2018, 42 (july-diciembre) https://www.scielo.sa.cr/pdf/edu/v42n2/2215-2644-edu-42-02-00629.pdf

Frade, L. (2006). Desarrollo de competencias en educación básica: desde preescolar hasta secundaria. Calidad educativa consultores S. C. México, D. F.

Guevara, Y. y Guerra, J. (2021). Comprensión lectora en estudiantes universitarios. https://southfloridapublishing.com/ojs/index.php/jhea/article/view/494/454

Latorre, A. (2005). La investigación-acción. Conocer y cambiar la práctica educativa. Editorial Graó. Barcelona, España. https://www.uv.mx/rmipe/files/2019/07/La-investigacion-accion-conocer-y-cambiarla-practica-educativa.pdf

Marcus, B. (2014). Los datos visuales en investigación cualitativa. Ediciones Morata, S. L. Madrid SEP (2018). Planes de estudio 2018. DGESuM. CEVIE, Cd. De México, México. https://www.ceviedgesum.com/index.php/planes-de-estudios-2018/124 